\title{
Does the stigma of mental illness contribute to suicidality?
}

\author{
Nicolas Rüsch, Alina Zlati, Georgia Black and Graham Thornicroft
}

\author{
Summary \\ Mental disorders are associated with suicidality and \\ with stigma. Many consequences of stigma, such \\ as social isolation, unemployment, hopelessness \\ or stress, are risk factors for suicidality. Research is \\ needed on the link between stigma and suicidality as
}

well as on anti-stigma interventions and their effects on suicidality.

\section{Declaration of interest}

None.
Nicolas Rüsch (pictured) is a consultant psychiatrist and Professor of Public Mental Health at the Department of Psychiatry II at the University of UIm, Germany. Alina Zlati is executive director of Open Minds - Center for Menta Health Research and a PhD candidate at the Department of Sociology, Babes-Bolyai University, Cluj-Napoca, Romania. Georgia Black is a qualitative research associate in the Department of Applied Health Research at University College London, UK. Graham Thornicroft is a consultant psychiatrist at the South London \& Maudsley NHS Foundation Trust and Professor of Community Psychiatry at King's College London, Institute of Psychiatry.

Suicide is a leading cause of premature death and disease burden worldwide. ${ }^{1}$ Although suicidality is often a direct consequence of mental disorders and their symptoms, ${ }^{2}$ people with mental illness face a two-fold problem. Besides their symptoms, they belong to a stigmatised group ${ }^{3}$ and experience frequent discrimination in their daily lives. ${ }^{4}$ Here we discuss the stigma and discrimination associated with mental illness as modifiable factors that may contribute to suicidality, but can be targeted by interventions. The link between stigma and suicidality is well known for other stigmatised groups, for example individuals from ethnic or sexual minorities, ${ }^{5}$ but has not received the same research attention for people with mental illness.

\section{Consequences of stigma and discrimination}

Three main types of stigma can be distinguished that may contribute to suicidality. First, public stigma occurs when members of the general public endorse negative stereotypes and discriminate against people with mental illness; typical consequences of public stigma are social isolation and impaired social networks when members of the public distance themselves from people labelled as mentally ill. Public stigma can lead to unemployment if employers endorse negative stereotypes and can affect several other domains such as education and housing. Stigma and discrimination are typically experienced as social defeat, which in turn is associated with suicidality. ${ }^{6}$ Second, societal regulations can systematically disadvantage people with mental illness, which is called structural discrimination; for example, relatively poorer funding of mental health services as compared with physical health services can lead to poorer quality of care and reduced access to mental health services.

Third, self-stigma refers to people with mental illness who internalise negative stereotypes, leading to shame, social withdrawal and demoralisation. As a consequence of self-stigma individuals with mental illness may feel they are not worthy or able to pursue their life goals ('why try'). ${ }^{7}$ Two additional consequences of experiencing stigma are important in this context. One is hopelessness or the feeling that one's situation will not improve. Individuals with mental illness who hold their own group in low regard - conceptually similar to self-stigma - are more likely to report hopelessness after adjusting for depressive symptoms. ${ }^{8}$ Research has shown that, as a last stage of coping with ostracism, individuals can enter a resignation stage that is dominated by hopelessness. ${ }^{9}$ A second potential consequence of facing stigma is the cognitive appraisal of stigma as a stressor or the perception of stigma as a threat that exceeds one's coping resources. Increased stigma stress is associated with social anxiety, shame and hopelessness. ${ }^{10}$ Finally, all three forms of stigma can be barriers to help-seeking for mental health problems. ${ }^{11}$

\section{Risk factors and models of suicidality}

It follows from the previous paragraphs that many consequences of stigma and discrimination are also factors associated with suicidality. ${ }^{12,13}$ Although a comprehensive discussion of suicide models is beyond the scope of this paper, two conceptualisations are pertinent here: interpersonal and stress-diathesis models. First, the interpersonal theory of suicide posits that suicidality stems from a combination of perceived social isolation, or thwarted belongingness, and perceived burdensomeness, or the perception that one is a burden on others. ${ }^{14}$ This is consistent with stigma as a risk factor, since public stigma typically leads to social isolation; perceived burdensomeness can be understood as a facet of self-stigma when the person with mental illness has internalised negative stereotypes. Second, according to a stress-diathesis model of suicidality, the co-occurrence of biological or psychological vulnerabilities and psychosocial stressors can trigger suicidality. ${ }^{15}$ Stigma is a stressor with negative effects already present during the at-risk stages of mental illness; ${ }^{16}$ stigma stress is associated with hopelessness ${ }^{10}$ and thus can be conceptualised as contributing to suicidality according to stress-diathesis models. Finally, low rates of help-seeking for mental disorders are associated with higher suicide rates. ${ }^{1}$

\section{Stigma and suicidality - empirical findings}

There is an emerging literature to support the hypothesis that stigma variables contribute to suicidality. ${ }^{17}$ First, in population studies, shame and self-stigma were lower in regions with fewer suicides as compared with high suicide rate regions, ${ }^{18}$ and public stigma levels were inversely related to suicide rates in different countries across Europe. ${ }^{19}$ In a qualitative study among people 
with mental illness and a history of suicidal crises, a majority reported that stigma had contributed to their feeling at their worst. ${ }^{20}$ Quantitative studies found significant associations of suicidality with self-stigma among people with schizophrenia even after controlling for symptom levels, ${ }^{21}$ with shame, a proxy of selfstigma, among military personnel, ${ }^{22}$ and with stigma stress among secondary mental health service users (details available from the authors on request). Suicide rates are higher when appropriate treatments are not available; ${ }^{23}$ as mentioned above, reduced access to mental healthcare can be considered an index of structural discrimination, and stigma in general is a barrier to help-seeking. ${ }^{11}$

Nevertheless, the existing evidence is sketchy for several reasons. First, most studies that examined the link between stigma variables and suicidality were cross-sectional and thus cannot clarify the direction of causality between symptoms, suicidality and stigma variables; second, as a relatively rare event suicide is hard to study empirically, and examining suicidal ideation as a proxy has its limitations; finally, empirical investigations should distinguish between the stigma of mental illness as a risk factor for suicidality (the topic of this editorial) on the one hand and the stigma of the suicidal act itself on the other hand. The latter stigma could be harmful (for example, because it could delay help-seeking) as well as helpful (for example, because it could deter individuals from suicide).

\section{Implications for research and interventions}

In terms of research, we need longitudinal studies to produce evidence for the hypothesised link between stigma variables and suicide risk. These investigations should be theory based and examine hypotheses derived from, for instance, the interpersonal theory or stress-diathesis model of suicidality. Different forms of experiencing and reacting to stigma and their impact on suicidality need to be taken into account: anticipated and experienced discrimination by others; self-stigma and shame; and consequences of structural stigma such as poor quality of and reduced access to mental health services. These studies should consider direct effects of stigma variables on suicidality as well as effects mediated by depressive symptoms that in turn may be triggered by social rejection. ${ }^{24}$ Finally, previous stigma research has mainly examined experiences of and reactions to stigma as trait variables, but future studies could use ambulatory assessments to capture situational or state variables and more immediate reactions, including the role of impulsivity.

The link between stigma and suicidality would have important implications for mental health policy and practice. In our view, though, we need thorough research first; on the basis of its findings, recommendations for policymakers and practitioners on how to tackle stigma and suicidality should be suggested later on. Such research will identify the most relevant consequences of stigma that are also risk factors for suicidality, informing interventions with the ultimate goal of reducing the number of suicides - a specific case of the more general agenda to address stigma and discrimination as barriers to prevention in mental health. ${ }^{25}$ There is initial evidence for the efficacy of interventions to reduce public stigma ${ }^{26}$ and self-stigma, ${ }^{27}$ including stigma stress. ${ }^{28}$ However, it is unclear whether these interventions reduce suicidality. At a population level, the effect of broad anti-stigma campaigns on suicide rates should be examined and likewise the effect of more targeted programmes to reduce public stigma among, for example, adolescents on suicide rates in this group. In clinical settings, interventions that reduce self-stigma, stigma stress and shame might successfully reduce suicidality. The effects of better funding for mental healthcare, an index of reduced structural discrimination, on suicide rates should be examined. Furthermore, there is an ongoing discussion whether the new social media are a remedy for or rather a cause of social isolation, ${ }^{29}$ and the internet as a potential pathway to increase social inclusion and to address stigma and suicidality should be investigated. Finally, increased help-seeking may mediate the effects of anti-stigma initiatives on suicidality.

In conclusion, we have initial evidence to speculate about stigma as a factor contributing to suicidality. Unlike other variables, stigma and discrimination - whether at the societal or individual level - are in principle modifiable risk factors. Therefore research on interventions in this domain is needed with the ultimate goal of reducing suicide rates.

Nicolas Rüsch, MD, Department of Psychiatry II, University of UIm, Germany and Health Service and Population Research Department, King's College London, Institute of Psychiatry, UK; Alina Zlati, MA, Open Minds - Center for Mental Health Research and Department of Sociology, Babes-Bolyai University, Cluj-Napoca, Romania; Georgia Black, PhD, Department of Applied Health Research, University College London, UK; Graham Thornicroft, MD, PhD, Health Service and Population Research Department, King's College London, Institute of Psychiatry, London, UK

Correspondence: Nicolas Rüsch, Section of Public Mental Health, Department of Psychiatry II, University of UIm, Parkstrasse 11, D-89073 UIm, Germany. Email: nicolas.ruesch@uni-ulm.de

First received 31 Jan 2014, final revision 20 May 2014, accepted 16 Jul 2014

\section{References}

1 World Health Organization. Public Health Action for the Prevention of Suicide: A Framework. WHO, 2012.

2 Cavanagh JT, Carson AJ, Sharpe M, Lawrie SM. Psychological autopsy studies of suicide: a systematic review. Psychol Med 2003; 33: 395-405.

3 Schomerus G, Schwahn C, Holzinger A, Corrigan PW, Grabe HJ, Carta MG, et al. Evolution of public attitudes about mental illness: a systematic review and meta-analysis. Acta Psychiatr Scand 2012; 125: 440-52.

4 Lasalvia A, Zoppei S, van Bortel T, Bonetto C, Cristofalo D, Wahlbeck K, et al. Global pattern of experienced and anticipated discrimination reported by people with major depressive disorder: a cross-sectional survey. Lancet 2013; 381: 55-62.

5 Goldston DB, Molock SD, Whitbeck LB, Murakami JL, Zayas LH, Hall GC. Cultural considerations in adolescent suicide prevention and psychosocial treatment. Am Psychol 2008; 63: 14-31.

6 Taylor PJ, Gooding P, Wood AM, Tarrier N. The role of defeat and entrapment in depression, anxiety, and suicide. Psychol Bull 2011; 137: 391-420.

7 Corrigan PW, Larson JE, Rüsch N. Self-stigma and the "why try" effect: impact on life goals and evidence-based practices. World Psychiatry 2009; 8: 75-81.

8 Rüsch N, Corrigan PW, Wassel A, Michaels P, Olschewski M, Wilkniss S, et al. Ingroup perception and responses to stigma among persons with mental illness. Acta Psychiatr Scand 2009; 120: 320-8.

9 Wesselmann ED, Williams KD. Ostracism and stages of coping. In The Oxford Handbook of Social Exclusion (ed CN DeWall): 20-30. Oxford University Press, 2013.

10 Rüsch N, Corrigan PW, Powell K, Rajah A, Olschewski M, Wilkniss S, et al. A stress-coping model of mental illness stigma: II. Emotional stress responses, coping behavior and outcome. Schizophr Res 2009; 110: 65-71.

11 Clement S, Schauman O, Graham T, Maggioni F, Evans-Lacko S, Bezborodovs $\mathrm{N}$, et al. What is the impact of mental-health related stigma on help-seeking? A systematic review of quantitative and qualitative studies. Psychol Med 2014; Feb 26: 1-17 (Epub ahead of print).

12 Pompili M, Serafini G, Innamorati M, Lester D, Shrivastava A, Girardi P, et al. Suicide risk in first episode psychosis: a selective review of the current literature. Schizophr Res 2011; 129: 1-11.

13 Wray M, Colen C, Pescosolido B. The sociology of suicide. Ann Rev Sociol 2011; 37: 505-28.

14 van Orden KA, Witte TK, Cukrowicz KC, Braithwaite SR, Selby EA, Joiner TE, Jr. The interpersonal theory of suicide. Psychol Rev 2010; 117: 575-600.

15 van Heeringen $\mathrm{K}$. Stress-diathesis model of suicidal behavior. In The Neurobiological Basis of Suicide (ed Y Dwivedi): 113-24. CRC Press, 2012. 
16 Rüsch N, Corrigan PW, Heekeren K, Theodoridou A, Dvorsky D, Metzler S et al. Well-being among persons at risk of psychosis: the role of self-labeling, shame and stigma stress. Psychiatr Serv 2014; 65: 483-9.

17 van Orden KA, Joiner TE. Depression and suicide: transactional relations with rejection. In The Oxford Handbook of Social Exclusion (ed CN DeWall): 211-9. Oxford University Press, 2013.

18 Reynders A, Kerkhof AJFM, Molenberghs G, van Audenhove C. Attitudes and stigma in relation to help-seeking intentions for psychological problems in low and high suicide rate regions. Soc Psychiatry Psychiatr Epidemiol 2014 49: 231-9.

19 Schomerus G, Evans-Lacko S, Rüsch N, Mojtabai R, Angermeyer MC, Thornicroft G. Collective levels of stigma and national suicide rates in 25 European countries. Epidemiol Psychiatr Sci 2014; Feb 27: 1-6 (Epub ahead of print).

20 Eagles JM, Carson DP, Begg A, Naji SA. Suicide prevention: a study of patients' views. Br J Psychiatry 2003; 182: 261-5.

21 Sharaf AY, Ossman LH, Lachine OA. A cross-sectional study of the relationships between illness insight, internalized stigma, and suicide risk in individuals with schizophrenia. Int J Nurs Stud 2012; 49: 1512-20.

22 Bryan CJ, Ray-Sannerud B, Morrow CE, Etienne N. Shame, pride, and suicidal ideation in a military clinical sample. J Affect Disord 2013; 147: 212-6.
23 Rihmer Z, Kiss K. Bipolar disorders and suicidal behaviour. Bipolar Disord 2002; 4 (suppl 1): s21-5.

24 Witvliet M, Brendgen M, van Lier PAC, Koot HM, Vitaro F. Early adolescent depressive symptoms: Prediction from clique isolation, loneliness, and perceived social acceptance. J Abnorm Child Psychol 2010; 38: 1045-56.

25 Rüsch N, Thornicroft G. Does stigma impair prevention of mental disorders? Br J Psychiatry 2014; 204: 249-51.

26 Corrigan PW, Morris SB, Michaels PJ, Rafacz JE, Rüsch N. Challenging the public stigma of mental illness: a meta-analysis of outcome studies. Psychiatr Serv 2012; 63: 963-73.

27 Mittal D, Sullivan G, Chekuri L, Allee E, Corrigan PW. Empirical studies of self-stigma reduction strategies: a critical review of the literature. Psychiatr Serv 2012; 63: 974-81.

28 Rüsch N, Abbruzzese E, Hagedorn E, Hartenhauer D, Kaufmann I, Curschellas J, et al. The efficacy of Coming Out Proud to reduce stigma's impact among people with mental illness: pilot randomised controlled trial. Br J Psychiatry 2014; 204: 391-7.

29 Parigi P, Henson WI. Social isolation in America. Ann Rev Sociol 2014; 40 : 153-71.

extra Intense romantic love in a 12th-century Persian medical encyclopaedia

\section{Behnam Dalfardi, Golnoush Sadat Mahmoudi Nezhad and Ahmad Ghanizadeh}

Hussain ibn Muhammad ibn Mahmoud ibn Ahmad Hussaini Jorjan (1042-1137 AD), known as Hakim Esmail Jorjani (Jurjani) in the west, was born in the Old Persian city of Gorgan (northeastern Iran). He studied medicine under the guidance of Abd al-Rahman ibn Ali ibn Abi Sadegh, who was often remembered as The Second Hippocrates.

At the age of 70, Jorjani wrote Zakhireh-i Kharazmshahi (Treasure of King Khwarazm), one of the first medical encyclopaedias written in the Persian language. This textbook was more than 750000 words long, comprised 10 volumes, and covered almost all aspects of medicine. It is the largest work on the healing arts ever compiled in the Persian language. In writing this text, in addition to his own extensive experience in medical science, Jorjani gathered the extant knowledge of over 30 of his predecessors.

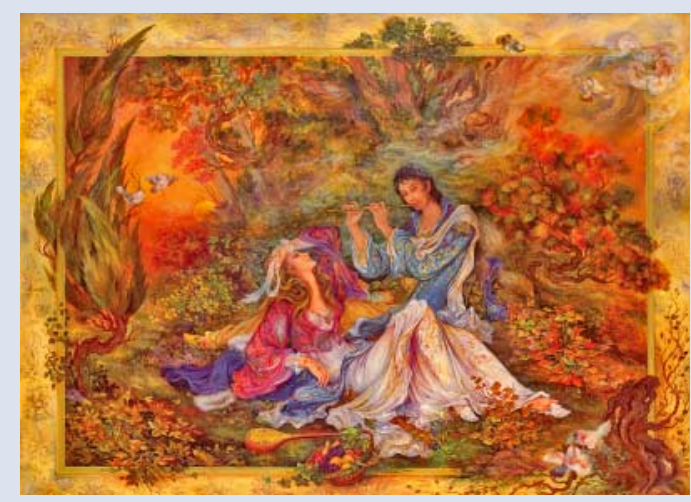

Enchanted Melody by Master Mahmoud Farshchian. 1987, USA A man playing a flute and the vibrant autumn colours excite a young woman's pleasure, and the young couple revel in each other's company. (Reproduced by permission from Master Mahmoud Farshchian).

Zakhireh provided early descriptions, particularly in volume 6, on issues that correspond to a number of present-day neuropsychiatric disorders and their manifestations. Among them is intense romantic love, a cross-cultural phenomenon with a special place in Old Persian writings. Jorjani defined intense romantic love as an obsession with components of depression, in which sexual desires play a role in establishment of the disorder. He explained that the victim of such an obsession shows heightened attention towards the object of his love. Jorjani characterised the beloved person as having sunken eyes, dryness of the eyes, as crying intermittently, and having disturbed sleep patterns. His speech is frequently accompanied by eye motions, smiles and repetitive sighs. The individual is delighted upon hearing poetry on the object of his love, and saddened when listening to a story on separation. The patient experiences an irregular pulse, especially when visiting the object of his affection, hearing her name or description. Jorjani primarily used the last feature to diagnose the condition.

Jorjani suggested ways to manage intense romantic love. He explained that patients with this affliction need physical and psychiatric supportive care (for example, to improve their diet and sleep and their hope for the future). He proposed occupying them in important professions to give them less time to think about their obsession. Another method he recommended was to introduce a possible new object of affection to such a patient and facilitate forgetting the previous object by admiring the new candidate's qualities. A third technique is for friends to wisely admonish the patient to change his ideas. The final recommendation is to enlist other women to discuss the bad character of the first love with the patient. He also explained that some patients require treatment for depression.

Jorjani's description of intense romantic love meets a number of symptoms mentioned in modern diagnostic criteria for some psychiatric disorders, including obsessive-compulsive disorder, separation anxiety disorder, adjustment disorder and major depressive disorder. He described a psychosomatic reaction as a main diagnostic test for this disorder (i.e. pulse rate change). His advised treatment methods represented an early form of cognitive-behavioural therapy and interpersonal psychotherapy. 